\title{
O066: Thermal disinfection of bedpans: European ISO 15883-3 guideline requirements are insufficient to ensure elimination of ARE and OXA-48 outbreak-strains
}

\author{
LB van der Velden ${ }^{1,2^{*}}$, MH Nabuurs-Franssen ${ }^{1}$, A van Leeuwen ${ }^{1}$, M Isken ${ }^{1}$, A Voss ${ }^{1,2}$ \\ From 2nd International Conference on Prevention and Infection Control (ICPIC 2013) \\ Geneva, Switzerland. 25-28 June 2013
}

\section{Introduction}

During 2012, our hospital was faced with a vancomycinresistant Enterococcus faecium (VRE) outbreak. Washerdisinfectors are used for the cleaning and thermal disinfection of bedpans. The European guideline NEN-ISO 15883-3 (ISO) states that washer- disinfectors have to achieve a minimum $\mathrm{A}_{0}$ value of 60 for appropriate disinfection of bedpans $\left(\approx 80{ }^{\circ} \mathrm{C}\right.$ for 60 seconds or $90{ }^{\circ} \mathrm{C}$ for 6 seconds). However, previous data have shown that some E. faecium strains survive 60 seconds at $80{ }^{\circ} \mathrm{C}$. Following ISO, the $\mathrm{A}_{0}$ measurement should include a cold (a minimum interval of 60 minutes since the machine was last used) and hot start.

\section{Objectives}

We determining the $A_{0}$ value of the VRE outbreakstrain and the outbreak-strain of the OXA-48 K.pneumoniae outbreak in another Dutch hospital during 2011. Moreover the impact of a cold start measurement on the $A_{0}$ value was evaluated.

\section{Methods}

The minimum $\mathrm{A}_{0}$ value that results in the killing of all isolates was determined for both strains. Bacterial suspensions were heated at 65,75 and $80{ }^{\circ} \mathrm{C}$ and samples for viable counts were obtained after $1,2,3$ and 10 minutes at each temperature. VRE PCR and cultures were performed on bedpan swabs after disinfection; hot and cold start measurements were compared.

${ }^{1}$ Medical Microbiology and Infectious Diseases, Canisius Wilhelmina Hospital Nijmegen, The Netherlands

Full list of author information is available at the end of the article

\section{Results}

Adequate killing required a minimum $\mathrm{A}_{0}$ value of 180 for the VRE outbreak strain and 120 for the OXA-48 K.pneumoniae. The cold start resulted in a $30 \%$ lower $\mathrm{A}_{0}$ value, than the hot start. All washer-disinfectors in our hospital functioned in agreement with the European guideline, although the lowest $A_{0}$ value was 73, only just above 60 . Swabs taken from bedpans processed in these washers with low $\mathrm{A}_{0}$ values, were VRE-positive by PCR and cultures.

\section{Conclusion}

Both outbreak strains survived the $A_{0}$ value of 60 required in the ISO. VRE were identified by PCR as well as cultures of bedpans that had been disinfected by these washer-disinfector. We suggest to increase the minimal acceptable $A_{0}$ value of washer-disinfectors to at least 180. Furthermore, the cold-start is needed for adequate $\mathrm{A}_{0}$-value measurement.

\section{Disclosure of interest}

None declared.

\section{Author details}

Medical Microbiology and Infectious Diseases, Canisius Wilhelmina Hospital Nijmegen, The Netherlands. ${ }^{2}$ Medical Microbiology, Radboud University Nijmegen Medical Center, Nijmegen, The Netherlands.

Published: 20 June 2013

doi:10.1186/2047-2994-2-S1-066

Cite this article as: van der Velden et al:: 0066: Thermal disinfection of bedpans: European ISO 15883-3 guideline requirements are insufficient to ensure elimination of ARE and OXA-48 outbreak-strains. Antimicrobial Resistance and Infection Control 2013 2(Suppl 1):O66. 